\title{
Misanthropic Person Memory When the Need to Self-Enhance Is Absent
}

\author{
Oscar Ybarra \\ University of Michigan
}

This research examined the role that the removal of the need or ability to self-enhance can play in the misanthropic processing of attributed behavioral information (i.e., remembering best negative, internally attributed behaviors and positive externally attributed behaviors). Experiment 1 demonstrated that removing a person's need to self-enhance by increasing his or her self-esteem eliminated misanthropic memory, whereas misanthropy was preserved for control participants and perceivers who had experienced a decrease in self-esteem. Furthermore, controlling for participants' self-evaluations eliminated the memory pattern differences between the two experimental conditions. Experiment 2 demonstrated that canceling the ability to self-enhance by having perceivers form an impression of themselves eliminated the misanthropy effect. However, the misanthropy effect was replicated when perceivers learned about an unknown other. The results were discussed with regard to the situations and factors that can increase or reduce the need to self-enhance and their implications for social information processing.

M emory for behavioral incidents accompanied by attributions can be characterized as misanthropic (Ybarra \& Stephan, 1996). People recall two types of attributed behaviors best: negative behaviors that are internally attributed and positive behaviors that are externally attributed. This pattern of recall is misanthropic because people preferentially recall behaviors that cast the target in the most negative light, that is, negative behaviors for which the target is blamed and positive behaviors for which the target is not given credit.

The misanthropy effect is important for various reasons. First, it demonstrates a bias in memory that creates negative perceptions of others (Ybarra, 1997). Second, it is consistent with research demonstrating negativity effects in social information processing (e.g., Anderson, 1965; Fiske, 1980; Hamilton, Dugan, \& Trolier, 1985; Hamilton \& Zanna, 1972; Klein, 1991; Pratto \& John, 1991; Skowronski \& Carlston, 1987; Wyer, 1970; Wyer \& Gordon, 1982, Experiment 2). However, an important set of questions that remains to be addressed concerns the conditions under which people will and will not display the misanthropy effect in memory. The present research is an attempt at delineating some of these boundary conditions by examining the role that self-enhancement plays in the processing of attributed behavioral information.

Recent research has extended the misanthropic memory findings to the perception of groups (Ybarra, Stephan, \& Schaberg, 1998). After being presented with attributed behavioral information about group members, perceivers showed better memory for negative behaviors that were internally attributed but positive behaviors that were externally attributed. Misanthropic memory for group members was not affected by the presence of group stereotypes or the knowledge that the group was cohesive or loosely knit. Subsequent research has shown that misanthropic group memory is affected by factors important in intergroup perception. In a second experiment, Ybarra et al. (1998) showed that the misanthropy effect was preserved when people learned about the out-group but that it was eliminated when people learned about the in-group. These findings are consistent with research showing the ultimate attribution error (Pettigrew, 1979), in which people attribute negative out-group and positive in-group behaviors to internal factors (i.e., the person) but positive out-group and negative in-group behaviors to external factors (i.e., the situation) (e.g., Bell, Wicklund, Manko, \& Larkin, 1976; Hewstone, 1990; Stephan, 1977). The Ybarra et al. findings are also concordant with intergroup memory research showing that perceivers recall more unfavorable than favorable traits about the out-group yet recall more favorable than unfavorable traits about the in-

Author's Note: I would like to thank James Hilton and Walter Stephan for their comments on an earlier version of this manuscript.

PSPB, Vol. 25 No. 2, February 1999 261-269

(C) 1999 by the Society for Personality and Social Psychology, Inc. 
group (Dutta, Kanungo, \& Freibergs, 1972) and more unfavorable behaviors about the out-group than the in-group (Howard \& Rothbart, 1980). As a whole, these attribution and memory findings support the notion that people are motivated to create a positive social identity from the groups to which they belong by ascribing negative qualities to the out-group and positive qualities to the in-group (e.g., Mackie, Hamilton, Susskind, \& Rosselli, 1996; Tajfel, 1978, 1982).

Not only do people strive to create positive social identities, they also strive to create positive individual identities (Baumeister, 1994; Steele, 1988; Tesser, Martin, \& Cornell, 1994). They can do so by ascribing negative qualities to others but not to the self. The misanthropy effect in memory for individual others may reflect the selective processing of others' behaviors in order to enhance and bolster a positive view of self. That is, by attending to and elaborating the negative internally attributed and positive externally attributed behaviors, perceivers can make favorable social comparisons (cf. Crocker, 1993; Wills, 1981; Wood, 1989; Wood, Taylor, \& Lichtman, 1985). An implication of this reasoning is that removing a person's need to self-enhance should eliminate the misanthropic processing of attributed behavioral information. One way this could be accomplished would be to allow perceivers to affirm themselves prior to processing information about a target.

Research has shown that perceivers who feel good about themselves due to succeeding at a task or receiving favorable personality feedback are relatively ineffective at processing negative information about others (e.g., Crocker, 1993; Forgas \& Bower, 1987). In contrast, perceivers who feel bad about themselves due to failing at a task or receiving unfavorable personality feedback are relatively effective at processing negative information about others (Crocker, 1993; Forgas \& Bower, 1987). This research coupled with the idea that people process information about others to self-enhance, converge on the suggestion that if people are made to feel good about themselves they should show a reduced tendency to process attributed behavioral information misanthropically. It might also be expected that if made to feel bad about themselves, people should show an amplification of the misanthropy effect. However, given that the misanthropy effect already reflects a biased recall pattern, it may not be possible to increase the degree to which perceivers can process attributed behavioral information misanthropically, even though they might be motivated to do so.

In the first experiment, increases and decreases in self-esteem were manipulated via a success or failure experience on an analogical reasoning test. Participants were then directed to an ostensibly unrelated impression formation task, in which they learned about an unfamil- iar other who had engaged in different positive and negative behaviors that were attributed to either internal or external causes. A set of control participants who received no success or failure feedback served as a comparison group. Following the reasoning proposed by Ybarra and Stephan (1996) and the findings of related research (Crocker, 1993; Forgas \& Bower, 1987), it was expected that participants who experienced a success would show a decrease in the misanthropy effect because their need to self-enhance would be reduced. In contrast, it was thought that participants who experienced a failure might display an increased misanthropy effect.

\section{EXPERIMENT 1}

\section{Method}

Design and participants. Participants in the experiment were 90 students who were distributed randomly to either an increase in self-esteem, decrease in self-esteem, or control condition ( 30 per between-participants condition). All students were presented with positive and negative behaviors that were linked to explicit internal and external attributions. Thus, the design of the study was a 3 (increase in self-esteem, decrease in self-esteem, control) $\times 2$ (valence of behavior) $\times 2$ (attribution type) mixed design, with the first factor varying between participants and the latter two factors varying within participants.

Self-esteem manipulation. Temporary increases and decreases in self-esteem were induced by having participants complete a test of analogical reasoning. The manipulation involved having participants work on a set of analogies that were pilot tested to be easy (success, increase in self-esteem condition) or difficult (failure, reduction in self-esteem condition).

Pretesting was conducted on the analogical reasoning test to assess how long it would take participants to complete the test in both conditions and to assess participants' performance on the test. The pretesting showed that participants in the success condition required approximately 4 minutes to complete the test, whereas participants in the failure condition required approximately 5.5 minutes to complete the test. Because both self-esteem conditions were run simultaneously, participants were given 7 minutes to take the test to ensure that all of them had time to complete it. The performance on the pretest confirmed the difficulty of the two tests. Participants in the success conditions answered on average 19 of the 20 analogies correctly. Participants in the failure condition answered on average 8.75 of the 20 analogies correctly.

To reinforce the perception of success or failure on the analogical test, bogus national averages were provided as feedback. The averages were constructed in a 
manner that would guarantee that participants taking the easy test regarded their performances as successes and that participants taking the difficult test regarded their performances as failures.

Manipulation check of self-evaluation manipulation. In addition to responding to a question about their actual scores on the analogical reasoning test, participants were also asked to indicate their rank based on the national averages feedback they had previously been given. Participants were then given eight questions that assessed their self-evaluations with regard to their performance on the analogical reasoning test. Participants indicated on a 10-point response scale $(1=$ not at all to $10=$ extremely) the extent to which they felt confident, happy, disappointed, sad, competent, good, disapproving, and bad about their performance. The items were reverse scored where necessary.

Behavioral stimuli. The behaviors for the impression formation task consisted of 24 statements previously pilot tested and used by Ybarra and Stephan (1996). Twelve of the behaviors were positive in valence, and 12 were negative in valence. The 24 behavior statements were linked to attributions that referred to either the person's disposition (internal) or the situation (external) in which the behavior was performed. Examples included the following: "Bob was the first to ask the new employee over for dinner because he looks forward to entertaining" (positive-internal), "Bob returned the change at the supermarket because the customer behind him noticed the mistake" (positive-external), "Bob was fired from a job when he pretended to get hurt because he wanted to get some sick leave" (negative-internal), and "Bob looked at and tried to memorize the exposed test answers because there was much pressure to do well" (negative-external).

This original stimulus set was extensively pilot tested to ensure that no biases existed with regard to the behaviors' valence (positivity and negativity) and equivalence across the types of attributions (dispositional and situational). In addition, the behaviors were pilot tested to ensure that the different attributions' locus of causality (dispositionality and situationality) was equivalent across the differently valenced behaviors (positive and negative). To further ensure the generality of the stimulus materials, a second stimulus set was created for the present research by taking the original behavior stems (Ybarra \& Stephan, 1996) and linking them to attributions opposite to the ones they were assigned in the original stimulus set (e.g., if a dispositional attribution was employed in the original stimulus set, a situational attribution was employed in the second set). Because the equivalence of the valence (positivity and negativity) across the different attributions had been established for the behavior stems in the earlier research, only the equivalence of locus of causality was assessed for this new stimulus set (i.e., that the dispositional and situational attributions were equivalent across the positive and negative behaviors).

A set of norming participants $(n=16)$ rated the new stimulus set for locus of causality. These judgments were made on a 10-point scale that was anchored by 0 (very external, situational) and 9 (very internal, dispositional). The locus of causality ratings were then analyzed using a 2 (valence of behavior) $\times 2$ (attribution type) withinparticipants ANOVA. The analysis produced only one significant effect, a main effect for attribution type, $F(1$, $14)=89.98, p<.0001$. As expected, internally attributed behaviors were rated as more dispositional than externally attributed behaviors ( $M=7.62$ vs. $M=3.00$ ). Therefore, in the new stimulus set, as in the original, internally attributed behaviors were equally dispositional and externally attributed behaviors were equally situational, regardless of the valence of the behavior.

The behavioral stimuli were combined in booklets that corresponded to four different presentation schemes. The presentation schemes were constrained so that each of the four types of behaviors (positive-internal, positive-external, negative-internal, negative-external) appeared once in each of six blocks.

Procedure. Participants were recruited to take part in an experiment on social information processing. On arriving at the lab, participants were told that, in addition to the impression formation task, they would be asked to complete other unrelated tasks. For the first task, participants were told that they were going to help establish local norms for materials to be used in an analogical reasoning study the following semester. At this point, participants were administered the self-esteem manipulation via the analogical reasoning task. After participants completed the self-esteem manipulation, they were given the correct answers so that they could score their tests. Once they scored their tests and tallied the number of correct responses, the experimenter provided the participants with bogus national averages. The success participants were told they had performed in the top $5 \%$, whereas failure participants were told that they had performed at the 30th to 39th percentile. Because there were differences in the actual scores on the analogical test within the two conditions, the feedback percentiles were designed to be flexible.

Following the feedback phase of the self-esteem manipulation, participants were told that it is common practice when norming such tests to allow for a delay between taking the test and getting their reactions and comments on it. In the meantime, they were introduced to the impression formation task. Participants were told that they would be forming an impression of a person 
named Bob by reviewing behaviors Bob had performed in the past. Participants were presented with 1 of the sets of 24 behaviors. The behaviors were presented at 8-second intervals as participants developed their impressions. Following the impression formation task, participants were given a 5-minute interpolated task that consisted of labeling a map of the United States to reduce working memory influences on recall.

A surprise recall task followed the interpolated task. Participants were asked to recall and write down as many of the behaviors as they could from those that were presented to them during the impression-formation phase. They were also told that if they could not remember the exact wording, they could write down the idea. They were given 8 minutes to complete the recall task. After completing the recall task, participants were asked to answer the manipulation check items and were then thoroughly debriefed.

\section{RESULTS}

\section{Self-Evaluation Manipulation Checks}

To assess the effectiveness of the self-esteem manipulation, examinations were made of participants' actual performance on the analogical reasoning test, their selfreported ranks based on their test performance and the bogus national averages, and the eight-item scale designed to tap their self-evaluations. All three measures were analyzed independently with one-way ANOVAs with self-esteem condition (failure, success) as the independent variable. (These analyses only included the success and failure conditions because these dependent measures could not be assessed in the control condition.)

All three manipulation check measures indicated that the self-esteem manipulation was effective. The test scores indicated that participants in the success condition $(M=19.41)$ did better on the test than participants in the failure condition $(M=10.28), F(1,58)=589.21$, $p<.0001$. Participants in the success conditions also indicated that their ranking based on their test performance was much higher $(M=9.87)$ than participants in the failure condition $(M=4.93), F(1,58)=662.56, p<$ .0001 . Finally, the responses to the self-evaluation items $($ Cronbach's alpha $=.94)$ indicated that participants in the success condition evaluated themselves more positively $(M=7.87)$ than did participants in the failure condition $(M=4.60), \mathrm{F}(1,58)=111.74, p<.0001$. Furthermore, a check on participants' scoring of their tests indicated that a number of failure but no success participants made errors in scoring in a self-enhancing direction. In sum, success participants did better on the analogical reasoning test than did failure participants, and they also knew that their performance represented
TABLE 1: Overall Recall of the Differently Attributed Positive and Negative Behaviors Across Self-Evaluation Conditions

\begin{tabular}{lcc}
\hline & \multicolumn{2}{c}{ Attribution Type } \\
\cline { 2 - 3 } Behavior Valence & Dispositional & Situational \\
\hline Control condition & 1.23 & \\
$\quad$ Positive & 2.12 & 2.18 \\
$\quad$ Negative & 1.67 \\
Success condition & 1.28 & \\
$\quad$ Positive & & 1.63 \\
$\quad$ Negative & 1.23 & 2.17 \\
Failure condition & 2.42 & 2.28 \\
$\quad$ Positive & & 1.95 \\
$\quad$ Negative & & \\
\hline
\end{tabular}

a success, whereas failure participants knew their performance represented a failure.

\section{Recall}

Participants were given credit for correctly recalling a behavior if they produced the gist of the originally presented item-both the behavior and the associated attribution. Half credit was given if participants recalled a behavior stem without the attribution, but no credit was given if an attribution was recalled without its designated behavior stem. ${ }^{1}$ Participants' recall was submitted to a 3 (increase in self-esteem, decrease in self-esteem, control) $\times 2$ (valence of behavior) $\times 2$ (attribution type) mixed design, with repeated measures on the latter two factors. The analysis yielded the interaction of valence and attribution type that characterizes the misanthropy effect, $F(1,87)=10.31, p<.001$. However, the analysis also yielded the predicted three-way interaction of selfesteem condition, valence of behavior, and attribution type, $F(2,87)=9.22, p<.0002$. Table 1 contains the recall means for the differently attributed positive and negative behaviors across the success, control, and failure conditions. For ease of exposition, the different behaviors were classified as misanthropic (negative-internal and positive-external) or nonmisanthropic (negative-external and positive-internal). Simple effects analyses indicated that people in the control condition recalled more misanthropic than nonmisanthropic behaviors ( $M$ difference $=1.40), F(1,29)=10.35, p<.003$. In contrast, people in the success condition recalled more nonmisanthropic than misanthropic behaviors ( $M$ difference = -.67), $F(1,29)=3.46, p<.03$. The difference in differences is reliable, $F(1,58)=13.45, p<.0005$.

The recall pattern in the failure condition also differed from the success condition. People in the failure condition recalled more misanthropic than nonmisanthropic behaviors $(M$ difference $=1.52), F(1,29)=13.28$, $p<.001$. Compared to the recall pattern of the success condition described above ( $M$ difference $=-.67$ ), the 
difference in differences is reliable, $F(1,58)=15.80, p<$ .0002 . Thus, as expected, a success induction eliminated the misanthropic processing of attributed behaviors relative to the control and failure conditions. The failure condition did not increase the misanthropy effect. The difference in the recall of misanthropic and nonmisanthropic behaviors was equivalent between the control and failure conditions, $F(1.58)<1.00 .^{2}$

Although the memory patterns are consistent with the notion that removing a person's need to self-enhance should eliminate the misanthropic processing of attributed behavioral information, it is of interest to examine the memory patterns when controlling for participants' self-evaluations. If the degree of participants' need to self-enhance underlies the extent of misanthropic information processing, controlling for participants' self-evaluations should reduce the interaction of behavior type and self-esteem condition. Participants' recall was submitted to a 2 (self-esteem condition: failure, success) $\times 2$ (behavior type: misanthropic, nonmisanthropic) mixed design ANCOVA, controlling for selfevaluations (composite index of how confident, happy, disappointed, sad, competent, good, disapproving, and bad they felt about their performance). Compared to the original analysis, which showed a strong interaction indicating that the recall of misanthropic and nonmisanthropic behaviors depended on participants' self-esteem condition, $F(1,58)=15.80, p<.0002$, in controlling for participants' self-evaluations, the ANCOVA resulted in a substantially diminished interaction, $F(1,57)=5.01$, $p<.03$. More important, the reliable differences within the self-esteem condition were eliminated. Participants in the failure condition no longer recalled more misanthropic than nonmisanthropic behaviors, $F(1,28)=<$ 1.00 , and participants in the success condition no longer recalled more nonmisanthropic than misanthropic behaviors, $F(1,28)=<1.00$. This analysis provides additional evidence that people's need to self-enhance, or lack thereof, underlies the misanthropic processing of attributed behavioral information.

Examination of the overall analysis indicated that there was also a main effect for valence, $F(1,87)=8.13$, $p<.005$, and a main effect for attribution type, $F(1,87)=$ $10.71, p<.001$. The former main effect indicated that participants recalled more negatively valenced $(M=$ 3.87) than positively valenced behaviors $(M=3.33)$, whereas the latter main effect indicated that externally attributed behaviors $(M=3.96)$ were better recalled than internally attributed behaviors $(M=3.23)$.

\section{DISCUSSION}

The first experiment was conducted to examine the effect that increases and decreases in self-esteem would have on the processing of attributed positive and nega- tive behaviors. Consistent with the hypothesis posited by Ybarra and Stephan (1996) concerning the cause of the misanthropy effect, the findings indicated that reducing a perceiver's need to self-enhance resulted in the elimination of the misanthropy effect. Control participants and failure participants, by contrast, produced a replication of the misanthropy pattern, displaying a memory bias for negative internally attributed and positive externally attributed behaviors. Furthermore, controlling for participants' self-evaluations eliminated the interaction of behavior type and self-esteem condition, thus providing additional evidence for the self-enhancement hypothesis.

\section{EXPERIMENT 2}

The findings of Experiment 1 indicate that reducing a perceiver's need to self-enhance by increasing his or her self-esteem eliminates the misanthropic processing of attributed behavioral information. Another method of eliminating self-enhancement might be to deny people the opportunity to self-enhance. Just as people do not process information about the in-group misanthropically (Dutta et al., 1972; Howard \& Rothbart, 1980; Ybarra, Stephan, \& Schaberg 1998), it might be that processing attributed behavioral information about the self would undercut the misanthropy effect. That is, perceivers should not be able to self-enhance by processing attributed behavioral information about the self misanthropically. Recent research is consistent with these ideas. For example, Baumeister and Cairns (1992) have shown that when given personality feedback, people attend to it less if it is unfavorable rather than favorable. Perceivers have also been shown to be poor at recalling information about the self if it is negative and related to personal failure (Kuiper \& MacDonald, 1982; Silverman, 1964).

The second experiment sought to determine the influence that learning attributed behavioral information about the self would have on the recall of the information. Participants took part in an impression-formation task, in which they either learned about an unfamiliar other or were asked to imagine themselves as the instigator of different positive and negative behaviors that were attributed to either internal or external causes. It was reasoned that when participants were asked to form an impression of the self, it would be less likely that they would process attributed behaviors misanthropically because such processing could not have positive implications for self. In contrast, it was expected that the misanthropy effect would be replicated when people learned about another person (cf. Ybarra \& Stephan, 1996) because such processing can have positive implications for self. 


\section{Method}

Design and participants. This experiment used a 2 (target: self, other) $\times 2$ (behavior valence: positive, negative) $\times 2$ (attribution type: internal, external) mixed design, the first factor varying between participants and the latter two factors varying within participants. Given course credit for their participation were 46 randomly assigned participants ( $n=23$ per between-participant condition).

Target manipulation. For this manipulation, half of the participants were told that they would be forming an impression of a person named Bob. The other half of the participants were told that they were to form an impression of themselves. The instructions were as follows:

One of the things that psychologists are interested in is the way in which we observe and think about different types of people including ourselves. This involves many psychological processes and the experimental session you will participate in today is concerned with several of these.

You will be presented with a list of behaviors. (You are to imagine that you are the one who performed these behaviors). Your task is to read through the behaviors and attempt to form a coherent impression of Bob (yourself) based on the behaviors he (you) performed.

Behavioral stimuli and procedure. The behavioral stimuli used in this experiment were taken from the first experiment save for minor modifications. Specifically, for people forming an impression of themselves, each behavior began with the word I. For example, "I did so and so because." The presentation schemes were the same as those in the first experiment. Participants were recruited to take part in an experiment on social information processing. On arriving at the lab, participants were introduced to the task by reading a sheet of paper with preliminary instructions about forming impressions, which also included the target (self, other) manipulation. After reading through the sheet, participants were presented with the different behaviors. The remaining aspects of the procedure were the same as Experiment 1.

\section{Results}

The same recall scoring procedure used in Experiment 1 was used in this experiment. Participants' recall was then submitted to a 2 (target: self, other) $\times 2$ (behavior valence: positive, negative) $\times 2$ (attribution type: internal, external) mixed design ANOVA, with repeated measures on the latter two factors. The overall analysis yielded the interaction of valence of behavior and attribution type that characterizes the misanthropy effect, $F(1,44)=7.99, p<.007$. However, it also yielded a three-way interaction, $F(1,44)=9.54, p<.003$, generally indicating that the misanthropy pattern depended on
TABLE 2: Overall Recall of the Differently Attributed Positive and Negative Behaviors Across Target Conditions

\begin{tabular}{lcc}
\hline & \multicolumn{2}{c}{ Attribution Type } \\
\cline { 2 - 3 } Behavior Valence & Dispositional & Situational \\
\hline Other condition & 1.46 & 2.19 \\
$\quad$ Positive & 2.08 & 1.48 \\
$\quad$ Negative & & \\
Self condition & 1.60 & 1.83 \\
Positive & 1.46 & 1.73 \\
Negative & \\
\hline
\end{tabular}

whether they had formed an impression of themselves or somebody else. Table 2 contains the recall means for the differently attributed positive and negative behaviors across the between-participants conditions. Similar to Experiment 1, for ease of exposition, the different behaviors were classified as misanthropic (negative-internal and positive-external) or nonmisanthropic (negative-external and positive-internal). Simple effects analyses indicated that people in the control condition recalled more misan thropic than nonmisanthropic behaviors ( $M$ difference $=1.48), F(1,22)=17.77, p<.0004$. In contrast, people in the success condition recalled both types of behaviors equivalently ( $M$ difference $=-.06$ ), $F(1,22)<$ 1.00. Thus, the misanthropy effect was replicated when people learned about another person, but the effect was eliminated when the attributed behaviors were imagined to be about the self.

\section{GENERAL DISCUSSION}

The present research sought to delineate some of the conditions under which people will process attributed behavioral information in a misanthropic manner. The findings showed that people who experienced a success did not display the misan thropic memory effect. That is, they did not recall negative internally attributed and positive externally attributed behaviors better than negative externally attributed and positive internally attributed behaviors. Likewise, the misanthropy effect was also eliminated when people processed attributed behavioral information about the self.

An explanation for the findings of Experiment 1 is that people in the success condition, because of their increased self-esteem, did not have a need to self-enhance. Removing the need to self-enhance eliminated the utility of selectively processing and storing negative information about the target. Control and failure participants displayed the misanthropy effect because the need to self-enhance was still active in these participants. It might be suggested that positive affect in the success condition and negative affect in the failure condition were the outcomes of the experimental manipulations and that this might account for the pattern of memory 
results. If affect were the medium through which the target information was processed, two outcomes might be expected. First, it might be expected that positive affect would guide information processing in a moodcongruent manner (Bower, 1981). If this were the case, then the most positive of the behavioral information (positive, internally attributed) might enjoy an advantage in memory. However, positive, internally attributed behaviors were the worst recalled in the success condition. Second, it might be expected that the experience of positive affect might have resulted in a reduced capacity for elaborating information, whereas the experience of negative affect might have resulted in extensive information elaboration (see Clore, Schwarz, \& Conway, 1994, for a review). Such divergent approaches to the information would have resulted in a main effect for overall recall, with the failure condition producing higher recall than the success condition. However, there were no differences in overall recall. Thus, the findings of Experiment 1 appear to be more consistent with a motivational view than with predictions based on mood effects.

In Experiment 2, perceivers who were presented attributed behavioral information about the self did not show the misanthropy effect in memory. Misanthropic processing under such circumstances has no positive implications for self. If anything, misanthropic processing of self-related information might result in an informational threat to the self (Baumeister \& Cairns, 1992). When learning about an unfamiliar other, the misanthropy effect occurs because such information processing does have positive implications for the self by helping people to create a positive individual identity (Ybarra \& Stephan, 1996) in a manner that is similar to the way processing negative information about out-groups helps to facilitate the creation of positive social identities (Dutta et al., 1972; Howard \& Rothbart, 1980; Mackie et al., 1996; Tajfel, 1978, 1982; Ybarra, Stephan, \& Schaberg, 1998).

An alternative explanation for the findings of Experiment 2 is that perceivers may have found the processing task difficult to perform in the self condition. Having to imagine that they were the person who had enacted the different behaviors may have required extensive cognitive resources on the part of the participants. This difficulty could thus underlie the lack of misanthropy in the self condition. If task difficulty underlies the absence of misanthropy under this condition, differences in overall recall might be expected, with the self condition producing lower recall than the other target condition. However, there were no differences in overall recall. Thus, the findings of Experiment 2 are consistent with the view that processing information about the self eliminated the misanthropic processing of attributed behavioral information.

It is interesting to note that the reduction of misanthropic memory in both experiments resulted, for the most part, in the equivalent recall of all types of information. There was one reliable reversal in Experiment 1 showing the better memory of negative externally attributed behaviors when perceivers had experienced an increase in self-esteem. This pattern of reduced misanthropy and not its reversal was also obtained for the in-group in the experiment by Ybarra, Stephan, and Schaberg (1998). When people explain their own behaviors or the behaviors of in-group members, they tend to explain positive acts as arising from internal factors but negative acts as the result of situational factors (Bell et al., 1976; Hewstone, 1990; Pettigrew, 1979; Snyder, Stephan, \& Rosenfield, 1978; Stephan, 1977). Given this attributional egotism and in-group favoritism, it might have been expected that in addition to a reduction in misanthropy for self and in-group, there might have been an amplification in the processing of nonmisanthropic behaviors. This should be expected because the knowledge structures and processes that allow people to engage in attributional egotism might facilitate the processing of the more favorable behaviors. Instead, perceivers learning about the self or the in-group (Ybarra, Stephan, \& Schaberg, 1998) appeared to process the available information in a manner free from biases.

The absence of self-serving or in-group biases in the recall of attributed behavioral information may be due to various factors. First, when self-esteem is high, there is no need to self-enhance. With regard to Experiment 2 and the intergroup experiment (Ybarra, Stephan, \& Schaberg, 1998), when processing information about the self or the in-group, the information is processed against a backdrop of all of the other information the individual possesses about the self or the in-group. This information about the self and the in-group is likely to be quite varied (in-group heterogeneity). Furthermore, because information about self or in-group is not processed with the goal of making favorable social comparisons, the incoming information is simply assimilated and is not differentially processed for enhancement purposes. In contrast, when information is processed about others, it is the only information available to the perceiver. Similarly, information that is processed about the out-group is processed against a background of limited information. In both cases, information about others and out-groups has clear implications for social comparisons. Finally, it is known that people engage in self-enhancing attributions in success / failure studies where the relevance to the self is high (Snyder et al., 1978). It may be that the present studies do not elicit this motive when 
the information is about the imagined behavior of the self or when self-esteem is high.

In addition to self-enhancement, it is also possible that misanthropic information processing serves other motives. Emphasizing the bad in others may allow people to bolster their self views, but it can also be functional on a more general scale. According to such a perspective, social perception is guided by self-protection motives and the need to determine the safety of the environment (e.g., Peeters \& Czapinski, 1990; Pratto \& John, 1991). Thus, it is adaptive to assume the worst in others, perceiving negative behavior as arising from dispositional factors but not necessarily assuming that positive behavior reflects good dispositions (Ybarra, Schaberg, \& Keiper, 1998). However, when people are feeling good (e.g., Clore et al., 1994; Fiedler, 1988) or when people are learning about themselves, these self-protective motives may not be active. ${ }^{3}$ As a consequence, people in these circumstances may be less likely to elaborate misanthropic information when they encounter it. And given that the inactivity of the self-protective motive does not imply benevolent or generous information processing, there is no reason to expect that nonmisanthropic behaviors should enjoy a recall advantage over misanthropic behaviors. Thus, such an account also helps to explain the equivalent recall of all types of behaviors when people had experienced success or when they were learning about themselves. In addition, it helps to explain why misanthropy was not amplified in the failure condition in Experiment 2; for self-protection, all that matters is that the misanthropic behaviors are encoded and not that the perceiver ruminate over them.

Recent research in stereotyping and prejudice is consistent with the present results. Fein and Spencer (1997) have shown that perceivers will stereotype and negatively evaluate others after being threatened but not when the self has been affirmed. These investigators, however, have argued that derogation of others is likely only when perceivers have adequate knowledge of the stereotype of the out-group. The present findings (Experiment 1) suggest that knowledge of stereotypes is not a necessary condition for emphasizing the bad in others. Knowing that another engages in negative acts or possesses negative qualities may be enough to trigger self-enhancement processes.

The findings of the present research and those obtain by Ybarra, Stephan, and Schaberg (1998) indicate that the target of the impression has an impact on whether people process attributed behavioral information in a misanthropic manner. Targets that enable perceivers to draw favorable comparisons facilitate misanthropic information processing, whereas targets that do not allow the perceiver to draw favorable comparisons attenuate or eliminate misanthropy. However, the degree to which a target facilitates favorable comparisons on the part of the perceiver may depend on the dimension of comparison. It may be that different situations make different dimensions of comparison salient. An academic environment may make scholarship particularly salient, whereas a sports situation may make athletic ability salient. People's memories of others may thus be closely tied to the situations in which the others are perceived, with some situations promoting negative perceptions and memory such as misanthropy and other situations facilitating a less disparaging approach to thinking about others.

It may also be that people from different cultural traditions will process information about others differently. For example, Asian collectivists are less likely to engage in attributional egotism and attributions that favor the in-group compared to the out-group (Boski, 1983; Fry \& Ghosh, 1980; Heine \& Lehman, 1997; Kashima \& Triandis, 1986). As a consequence, perceivers from such cultures may have less of a need to create or enhance a positive view of self. This reduced need to create and maintain a positive image of self may serve to reduce misanthropic processing of attributed behavioral information.

In conclusion, an examination of the notion of selfenhancement in the processing of attributed behavioral information not only allows us to investigate the boundary conditions of the misanthropy effect, but it also suggests how different targets, different situations, and culture might bring about social cognition that is driven to a greater or lesser extent by the need to emphasize the bad in others.

\section{NOTES}

1. The scoring method was chosen for three reasons. First, person memory research has historically emphasized memory for behaviors. Second, a behavior recalled by itself is meaningful, whereas an attribution recalled by itself is not. The findings from Ybarra and Stephan (1996) attest to this reasoning. Their internal analyses of the recall data indicated that out of all of the behaviors recalled, $78 \%$ were recalled with the associated attribution, $18 \%$ without the associated attribution, and only $4 \%$ of the information recalled was composed of attributions without behaviors. These results suggest that attributions taken by themselves were not meaningful enough to be remembered. Finally, neither the recall of the behaviors without attributions nor the attributions without their behaviors coincided with the misanthropy pattern in the research by Ybarra and Stephan (1996).

2. It is possible that the lack of an amplification of the misanthropy effect for the failure condition might have resulted from one of two reasons, one methodological and one psychological. First, failure participants on average reported that they had performed at the 40 th to 49th percentile. Although anyone reading this article would regard these percentiles as clear failure feedback, maybe the participants viewed their performances as poor but not necessarily as profound failures. Alternatively, it may be the case that misanthropic person memory represents a ceiling effect; people have a tendency to engage in misanthropy, so it may be difficult to get them to engage in more misanthropy.

3. I thank the reviewers for this suggestion. 


\section{REFERENCES}

Anderson, N. H. (1965). Primary effects in personality impression formation using a generalized order effect paradigm. Journal of Personality and Social Psychology, 2, 1-9.

Baumeister, R. F. (1994). Self-regulation and ego threat: Motivated cognition, self-deception, and destructive goal setting. In P. M. Gollwitzer \& J. A. Bargh (Eds.), The psychology of action. New York: Guilford.

Baumeister, R. F., \& Cairns, K. J. (1992). Repression and self-presentation: When audiences interfere with self-deceptive strategies. Journal of Personality and Social Psychology, 62, 851-862.

Bell, L. G., Wicklund, R. A., Manko, G., \& Larkin, C. (1976). When unexpected behaviors is attributed to the environment. Journal of Research in Personality, 10, 316-327.

Boski, P. (1983). A study of person-perception in Nigeria: Ethnicity and self versus other attributions for achievement-related outcomes. Journal of Cross-Cultural Psychology, 14, 85-108.

Bower, G. (1981). Mood and memory. American Psychologist, 36, 129-145.

Clore, G. L., Schwarz, N., \& Conway, M. (1994). Affective causes and consequences of social information processing. In R. S. Wyer and T. K. Srull (Eds.), Handbook of social cognition: Vol. 1. Basic processes. Hillsdale. NJ: Lawrence Erlbaum.

Crocker, J. (1993). Memory for information about others: Effects of self-esteem and performance feedback. Journal of Research in Personality, 27, 35-48.

Dutta, S., Kanungo, R. N., \& Freibergs, V. (1972). Retention of affective material: Effects of intensity of affect on retrieval. Journal of Personality and Social Psychology, 23, 64-80.

Fein, S., \& Spencer, S. (1997). Prejudice as self-image maintenance: Affirming the self through derogating others. Journal of Personality and Social Psychology, 73, 31-44.

Fiedler, K. (1988). Emotional mood, cognitive style, and behavior regulation. In K. Fiedler \& J. Forgas (Eds.), Affect, cognition, and social behavior (pp. 100-119). Toronto, Canada: Hogrefe International

Fiske, S. T. (1980). Attention and weight in person perception: The impact of negative and extreme behavior. Journal of Personality and Social Psychology, 38, 889-906.

Forgas, J. P., \& Bower, G. H. (1987). Mood effects on person-perception judgments. Journal of Personality and Social Psychology, 53, 53-60.

Fry, P. S., \& Ghosh, R. (1980). Attributions of success and failure: Comparison of cultural differences between Asian and Caucasian children. Journal of Cross-Cultural Psychology, 11, 343-363.

Hamilton, D. L., Dugan, P. M., \& Trolier, T. K. (1985). The formation of stereotypic beliefs: Further evidence for distinctiveness-based illusory correlations. Journal of Personality and Social Psychology, 48, $5-17$.

Hamilton, D. L., \& Zanna, M. (1972). Differential weighting of favorable and unfavorable attributes in impressions of personality. Journal of Experimental Research in Personality, 6, 204-212.

Heine, S. J., \& Lehman, D. R. (1997). The cultural construction of self-enhancement: An examination of group-serving biases. Journal of Personality and Social Psychology, 72, 1268-1283.

Hewstone, M. (1990). The "ultimate attribution error"? A review of the literature on intergroup causal attribution. European Journal of Social Psychology, 20,311-335.

Howard, J. W., \& Rothbart, M. (1980). Social categorization and memory for in-group and out-group behavior. Journal of Personality $\mathcal{E}^{\circ}$ Social Psychology, 38, 301-310.

Kashima, Y., \& Triandis, H. C. (1986). The self-serving bias in attribution as a coping strategy: A cross-cultural study. Journal of Cross-Cultural Psychology, 17, 83-97.

Klein, J. G. (1991). Negativity effects in impression formation: A test in the political arena. Personality and Social Psychology Bulletin, 17, 412-418.

Kuiper, N. A., \& MacDonald, M. R. (1982). Self and other perception in mild depressives. Social Cognition, 1, 233-239.
Mackie, D. M., Hamilton, D. L., Susskind, J., \& Rosselli, F. (1996). Social psychological foundations of stereotype formation. In C. N. Macrae, C. Stangor, \& M. Hewstone (Eds.), Stereotypes and stereotyping. New York: Guilford.

Peeters, G., \& Czapinski, J. (1990). Positive-negative asymmetry in evaluations: The distinction between affective and informational negativity effects. In W. Stroebe \& M. Hewstone (Eds.), European review of social psychology, 11, 33-60. Wiley \& Sons.

Pettigrew, T. F. (1979). The ultimate attribution error: Extending Allport's cognitive analysis of prejudice. Personality and Social PSychology Bulletin, 5, 461-476.

Pratto, F., \& John, O. P. (1991). Automatic vigilance: the attention-grabbing power of negative social information. Journal of Personality and Social Psychology, 61, 380-391.

Silverman, I. (1964). Self-esteem and differential responsiveness to success and failure. Journal of Abnormal and Social Psychology, 69, 115-119.

Skowronski, J. J., \& Carlston, D. E. (1987). Social judgment and social memory: The role of cue diagnosticity in negativity, positivity, and extremity biases. Journal of Personality and Social Psychology, 52, 689699.

Snyder, M. L., Stephan, W. G., \& Rosenfield, D. (1978). Attributional egotism. In J. H. Harvey, W. Ickes, \& R. F. Kidd (Eds.), New directions in attribution research (Vol. 2). Hillsdale, NJ: Lawrence Erlbaum.

Steele, C. M. (1988). The psychology of self-affirmation: Sustaining the integrity of the self. In L. Berkowitz (Ed.), Advances in experimental social psychology (Vol., 21, pp. 261-302). New York: Academic Press.

Stephan, W. G. (1977). Stereotyping: the role of ingroup-outgroup differences in causal attribution for behavior. Journal of Social Psychology, 101, 255-266.

Tajfel, H. (1978). The achievement of group differentiation. In H. Tajfel (Ed.), Differentiation between social groups: Studies in the social psychology of intergroup relations. London: Academic Press.

Tajfel, H. (1982) . Social psychology of intergroup relations. In M. R. Rosenzweig \& L. W. Porter (Eds.), Annual review of psychology (Vol. 33, pp. 1-39). Palo Alto, CA: Annual Reviews.

Tesser, A., Martin, L. L., \& Cornell, D. P. (1994). On the substitutability of Self-Protective Mechanisms. In P. M. Gollwitzer \& J. A. Bargh (Eds.), The psychology of action. New York: Guilford.

Wills, T. A. (1981). Downward comparison principles in social psychology. Psychological Bulletin, 90, 245-271.

Wood, J. V. (1989). Theory and research concerning social comparisons of personal attributes. Psychological Bulletin, 106, 231-248.

Wood, J. V., Taylor, S. E., \& Lichtman, R. R. (1985). Social comparison in adjustment to breast cancer. Journal of Personality and Social Psychology, 49, 1169-1183.

Wyer, R. S. (1970). Information redundancy, inconsistency, and novelty and their role in impression formation. Journal of Experimental Social Psychology, 6, 111-127.

Wyer, R. S., \& Gordon, S. (1982). The recall of information about persons and groups. Journal of Experimental Social Psychology, 18, 128-164.

Ybarra, O. (1997). Unpublished raw data, University of Michigan, Ann Arbor.

Ybarra, O., Schaberg, L., \& Keiper, S. N. (1998). Target favorability and social information processing. Manuscript submitted for publication, University of Michigan, Ann Arbor.

Ybarra, O., \& Stephan, W. G. (1996). Misanthropic person memory. Journal of Personality and Social Psychology, 70, 691-700.

Ybarra, O., Stephan, W. G., \& Schaberg, L. (1998). Person memory for the attributed behavior of group members: From misanthropy to sexism. Manuscript submitted for publication, University of Michigan, Ann Arbor.

Received November 19, 1997

Revision Accepted March 1, 1998 